\title{
Effects of extracellular vesicles on placentation and pregnancy disorders
}

\author{
Changwon Yang ${ }^{1}$, Gwonhwa Song ${ }^{1}$ and Whasun Lim² \\ ${ }^{1}$ Institute of Animal Molecular Biotechnology and Department of Biotechnology, College of Life Sciences and \\ Biotechnology, Korea University, Seoul, Republic of Korea and ${ }^{2}$ Department of Food and Nutrition, Kookmin \\ University, Seoul, Republic of Korea
}

Correspondence should be addressed to G Song or W Lim; Email: ghsong@korea.ac.kr orwlim@kookmin.ac.kr

\begin{abstract}
In humans, pregnancy maintenance depends on normal placental formation following trophoblast invasion into the endometrium and vascular remodeling. In the early stages of pregnancy, immune tolerance, inflammatory response and adaptation to hypoxia need to be precisely regulated in the placental microenvironment. Various types of cells, such as trophoblasts, endothelial cells, immune cells, mesenchymal stem cells (MSCs) and adipocytes, induce normal placental development via intercellular interactions through soluble factors. Extracellular vesicles (EVs) are used to diagnose various diseases because their constituents vary depending on the type of cell of origin and pathological characteristics. EV-derived microRNAs (miRNAs) and proteins in the placenta regulate inflammatory responses and the invasion of trophoblasts through intercellular delivery in the placental microenvironment. If the placenta does not adapt to the changed environment during early pregnancy, pregnancy disorders such as pre-eclampsia, preterm birth and gestational diabetes mellitus can occur. Thus, the important roles of EVs during pregnancy and development is fast emerging. This review describes the physiological role of EVs during placentation and their composition in the human placenta. It also suggests the possibility of finding EV markers that can diagnose pregnancy disorders. Furthermore, it describes the properties of EVs that affect pregnancy in livestock.

Reproduction (2019) 158 R189-R196
\end{abstract}

\section{Introduction}

In humans, a successful pregnancy depends on the normal implantation and development of the placenta, which is a temporary organ responsible for fetal growth and development during the pregnancy period. In the fetomaternal interface during early pregnancy, an inner layer called the cytotrophoblast (CT) is attached to the basement membrane and an outer layer called the syncytiotrophoblast (ST) originates from the fusion of CT. ST is in direct contact with maternal blood and is responsible for the exchange of gas, nutrients and waste products between the mother and fetus. Several evidences show that the release of apoptotic debris from the ST causes a systemic inflammatory response. The villous trophoblasts (VTs) avoid immune system attacks by forming an immune privilege at the fetomaternal interface (Sargent et al. 2003). The extravillous trophoblasts (EVTs) penetrate the endometrium and the underlying myometrium to induce intimate interactions between the placenta and uterine wall. Another group of EVTs enter the uterine spiral arteries and induce remodeling of the blood vessels. During the first trimester, EVT invasion is controlled by cytokines and chemokines secreted by the decidual and immune cells. In the early stages of fetal implantation, the oxygen tension is $\sim 3 \%$ in the uterus and $8 \sim 12 \%$ in the decidua and myometrium (Jauniaux et al. 2003). In the fetomaternal interface, oxygen tension regulates EVT invasion and vascular remodeling in the myometrium.

Extracellular vesicles (EVs) are found in most body fluids, including blood, urine, saliva and breast milk and contain numerous types of RNAs, lipids and proteins. miRNA expression in trophoblast cells is reportedly altered in hypoxic and inflammatory placental environments. Not only are miRNAs abundant in EVs implicated in the pathological changes in diseases, but they can also stably exist in the circulatory system, which makes them potential biomarkers for diagnosing pregnancy disorders. Recent studies have shown that maternal obesity also contributes to the formation of pro-inflammatory environments and endothelial cell dysfunction in the placenta, which is related to the amount of maternal circulating EVs (Elfeky et al. 2017). Since EVs contain the metabolic products of cells, they make it possible to predict the physiological and pathological conditions of the cell of origin. Numerous trophoblast cell lines, such as JEG-3 choriocarcinoma and HTR8/SVneo EVT cell lines, have been used to elucidate the physiological effects of EVs. Furthermore, 
the placental explant culture model or perfusion methods have also helped to analyze the endothelial dysfunction mechanism using placenta-derived EVs (Gupta et al. 2008).

This review describes the effect of placentaderived $\mathrm{EVs}$ on placental immune response regulation and trophoblast cell characteristics in humans. The contribution of EVs to pregnancy disorders such as pre-eclampsia $(\mathrm{PE})$, preterm birth $(\mathrm{PB})$ and gestational diabetes mellitus (GDM) is also analyzed. Finally, the role of EVs in interactions between the conceptus and endometrium in livestock is described.

\section{Identification of placenta-derived EVs}

The characterization of EVs is not yet fully established. The components of the recovered EVs can vary depending on the extraction methods (Lotvall et al. 2014). In addition, extracellular RNA can be delivered through non-EV carriers, which further makes research on EVs challenging (Vickers et al. 2011). The exosome is the most widely studied $\mathrm{EV}$, but a method to accurately distinguish between exosomes has not been established. Numerous proteomic analyses of $\mathrm{EVs}$ have been conducted, but specific protein markers for each type of EV remain elusive.

Many studies have identified the composition of placental EVs derived from maternal circulation. Furthermore, the effect of EVs on placentation has been elucidated via in vitro studies by analyzing the changes in expression of miRNAs and proteins in EVs depending on the types and invasiveness of the trophoblast cells (Tong et al. 2016). The chromosome 19 miRNA cluster (C19MC), including miR-520c, is a highly expressed miRNA in placenta-derived EVs that changes its expression under hypoxic conditions and is likely to function in placental-maternal signaling (Donker et al. 2012).

Flow cytometry and nanoparticle tracking analysis (NTA) have not only enabled the detection of EVs in maternal circulation, but have also helped identify differences in the concentration and size of EVs between women with pregnancy disease and normal healthy women. Additionally, quantitative analysis of CD63, a typical EV marker, and placental alkaline phosphatase (ALPP), a placenta-specific marker, revealed that the concentration of placental EVs increased with the progress of pregnancy (Sarker et al. 2014). By injecting EVs labeled with fluorescent dyes into a rodent model, it was possible to image the delivery pattern of the EVs within the fetomaternal interface and other organs such as the lungs, liver and kidneys (Sheller-Miller et al. 2016, Tong et al. 2017). Engineered materials that can help analyze placenta-derived EVs more directly and sensitively have also been developed recently (Boriachek et al. 2019).

\section{Physiological functions of EVs in the placenta}

The functional activity of isolated EVs has been the focus of recent in vitro studies. However, investigation of physiological changes following delivery of EVs has been hindered by some experimental limitations (Lotvall et al. 2014). It is not easy to secure a treatment group capable of acting as a negative control in which EVs are depleted. It is also difficult to confirm the function of each EV subpopulation because various extraction methods are used. Since most of the studies that report the functional role of exosomes in the placenta have included these limitations, we have used the more general term 'EVs' rather than 'exosomes'.

Pregnancy is a condition characterized by mild immunosuppression and induced inflammation (Redman \& Sargent 2007). The activation of T cells along with the expression of inflammatory cytokines during pregnancy has been associated with the onset of pregnancy disorders such as PE and intrauterine growth retardation (IUGR). Moreover, during early pregnancy, the placenta is hypoxic, which increases endothelial cell proliferation and ultimately increases the surface area of the blood vessels, maximizing oxygen and nutrient transfer. By determining the number of ALPP-positive $E V s$, it is possible to investigate the effects of placental EVs in comparison to those of the total EV population in maternal blood (Table 1).

Trophoblasts express a number of immunoregulatory proteins that regulate maternal immune cell function (Petroff et al. 2005). EVs exhibit either immunostimulatory or immunosuppressive properties depending on their origin and composition. For instance, placental Fas ligand (FASLG) is released from the ST via EVs at the fetomaternal interface in order to promote an immune privilege status (Stenqvist et al. 2013). EVs carrying FASLG and CD274 inhibit T-cell activation signals such as those from janus kinase 3 (JAK3) and have immunomodulatory effects (Sabapatha et al. 2006). In addition to FASLG, TNF superfamily member 10 (TNFSF10) is also released from the ST via EVs, leading to apoptosis of T cells and/or peripheral blood mononuclear cells (PBMCs) (Stenqvist et al. 2013). Furthermore, EVs bearing soluble MHC class I chain-related molecules (MIC) and UL16-binding proteins (ULBP) inhibit the killer cell lectin like receptor K1 (KLRK1)-dependent cytotoxic responses of PBMCs and induce fetal immune escape during early pregnancy (Mincheva-Nilsson et al. 2006, Hedlund et al. 2009). Moreover, endogenous retrovirus group $\mathrm{W}$ member 1, envelope (ERVW-1) transfers to PBMCs through EVs and inhibits the response of lipopolysaccharide (LPS)/phytohemagglutinin (PHA)-induced cytokines. $\mathrm{C} 19 \mathrm{MC}$ in the EVs of trophoblast cells also induces viral resistance mediated by autophagy in nonplacental recipient cells (Delorme-Axford et al. 2013). Notably, miR-517a-3p is abundantly contained in EVs derived from trophoblast cells, is delivered to T cells and NK 
Table 1 Biological function of EVs in the placenta.

\begin{tabular}{|c|c|c|c|c|}
\hline $\begin{array}{l}\text { Active substance } \\
\text { in EVs }\end{array}$ & Recipient cells & Biological function & Target molecules/pathways & Reference \\
\hline MIC & PBMCs & Fetal immune escape & KLRK1 & Mincheva-Nilsson et al. 2006 \\
\hline FASLG, CD274 & T cells & T-cell suppression & - & Sabapatha et al. 2006 \\
\hline ULBP & NK cells, $T$ cells & Fetal immune escape & KLRK1 & Hedlund et al. 2009 \\
\hline- & Monocytes, B cells, PBMCs & Th2 cytokine release & - & Southcombe et al. 2011 \\
\hline ERVW-1 & PBMCs & Inhibition of Th1 cytokines & - & Tolosa et al. 2012 \\
\hline C19MC & Nonplacental cells & Viral resistance & Autophagy & Delorme-Axford et al. 2013 \\
\hline FASLG, TRAIL & T cells, PBMCs & Fetal immune escape & Apoptosis & Stenqvist et al. 2013 \\
\hline Fibronectin & Macrophages & Pro-inflammatory cytokine release & - & Atay et al. $2011 b$ \\
\hline- & Monocytes & Monocyte recruitment & - & Atay et al. 2011a \\
\hline miR-146a-3p & Trophoblast & IL-8 secretion & TLR8 & Gysler et al. 2016 \\
\hline- & Endothelial cells & Cytokine release & - & Elfeky et al. 2017 \\
\hline MiR-548c-5p & Macrophages & $\begin{array}{l}\text { Inhibition of inflammatory } \\
\text { cytokines }\end{array}$ & PTPRO & Wang et al. 2019 \\
\hline miR-520c-3p & EVTs & Increased invasion & CD44 & Takahashi et al. 2017 \\
\hline miR-155 & HUVECS & Inhibition of eNOS & - & Shen et al. 2018 \\
\hline
\end{tabular}

cells and is known to target the PRKG1 gene in recipient cells (Kambe et al. 2014).

Excessive pro-inflammatory effects in the placenta are associated with the onset of pregnancy disorders, which are characterized by systemic inflammation such as PE and PB. During pregnancy, macrophages are abundant in the maternal decidua and they regulate the inflammatory response at the fetomaternal interface by secreting various cytokines and chemokines. Fibronectin is transferred to macrophages via EVs derived from the trophoblast cells to promote the production of interleukin - $1 \beta$ (IL1B) (Atay et al. 2011b). In addition, EVs derived from trophoblasts increase the migration of monocytes, thus generating an inflammatory environment through the production of IL1B, IL6, SERPINE1, colony stimulating factor 2 (CSF2) (Atay et al. 2011a). The expression of miR-146a-3p in trophoblasts is increased in cell lysates and EVs via antiphospholipid antibodies, which promotes IL8 secretion through Toll-like receptor 8 (TLR8) activation (Gysler et al. 2016). On the contrary, miR-548c-5p, which is highly expressed in EVs derived from PE patients, inhibits the proliferation of macrophages and the expression of inflammatory cytokines such as IL12 and TNF (Wang et al. 2019). Maternal plasma EVs were also shown to cause inflammatory responses and PB in pregnant mice, which clarified the role of paracrine signaling in the progression of pregnancy inflammatory diseases (Sheller-Miller et al. 2019).

During the first trimester of pregnancy, placentaderived EVs also induce vasculogenesis and angiogenesis through an oxygen-sensing mechanism under hypoxic conditions. Proteomic analysis suggests that trophoblast EVs induce the activation of matrix metalloproteinases (MMPs) and mitogenactivated protein kinase (MAPK) signaling pathways. miR-520c-3p of EVs promotes the invasiveness of EVTs, which occurs by targeting CD44 in EVTs (Takahashi et al. 2017). Other C19MC miRNAs as well as miR-520c-3p are also speculated to regulate EVT migration. In addition, ST-derived EVs containing endothelial nitric oxide synthase (eNOS) facilitate the prediction of low nitric oxide (NO) biological activity in PE patients (Motta-Mejia et al. 2017). miR-155, which is highly expressed in the plasma and placenta of PE patients, is delivered to endothelial cells via EVs and inhibits the expression of eNOS (Shen et al. 2018). Thus, many miRNAs and proteins in placental EVs regulate the placental immune response and invasiveness of trophoblast cells. However, more research is needed regarding the genetic and epigenetic networks involved in the development of pregnancy disorders (Fig. 1).

The microenvironment around placental trophoblast cells has important physiological functions with respect to placental development. In the placental microenvironment, soluble factors secreted by various types of cells such as endometrial cells, mesenchymal stem cells (MSCs), adipocytes and macrophages play various roles during intercellular interactions in the fetomaternal interface. Several tracking experiments have shown that miR-30d and let-7a secreted from endometrial cells can be transferred to the trophoblast through EVs (Vilella et al. 2015, Niu et al. 2017). Moreover, endometrial EVs are internalized by trophoblast cells, which mediate focal adhesion kinase (FAK) signaling, thereby increasing their ability to attach to the endometrium (Greening et al. 2016). MSCs, which can be easily isolated in the placenta, contribute to the formation of the vascular system in the placenta by producing numerous pro-angiogenic factors such as vascular endothelial growth factor (VEGF), hepatocyte growth factor (HGF) and fibroblast growth factor2 (FGF2) (Kong et al. 2013). EVs derived from placental MSCs (pMSCs) promote migration and tube formation via placental microvascular endothelial cells in order to adapt to low oxygen tension. Moreover, pMSCs increase VEGF and miR-126 levels in the EVs and promote angiogenic processes in response to $\mathrm{NO}$ stimulation (Du et al. 2017). 


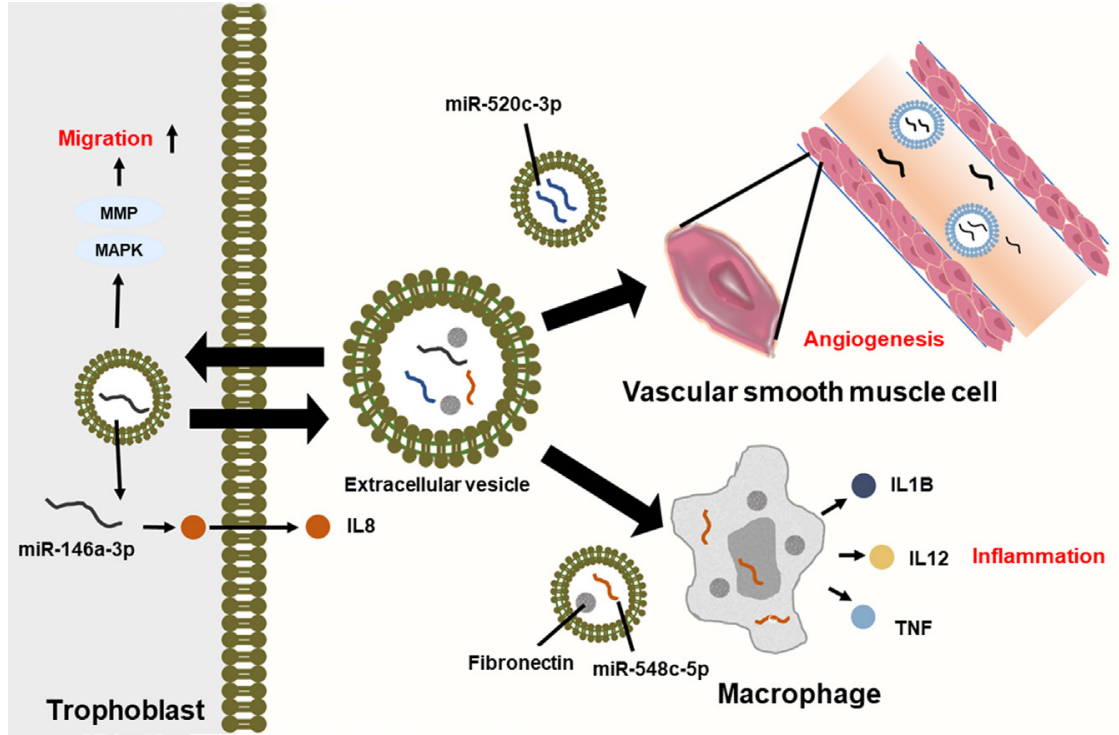

Figure 1 Effect of trophoblast-derived EVs on angiogenesis and inflammation. miRNAs and proteins in EVs control the migration of the trophoblast. An increase in the concentration of trophoblast EVs increases cell migration via the matrix metalloproteinase (MMP) and mitogen-activated protein kinase (MAPK) signaling pathways. Moreover, miR-146a-3p in EVs promotes the secretion of interleukin (IL)-8 from the trophoblast cells. EVs containing miR-520c-3p are also transferred to vascular smooth muscle cells (VSMCs) to increase cell migration and exhibit pro-angiogenic effects. Finally, trophoblast EVs internalized by macrophages promote cytokine secretion, including IL1B, IL12 and TNF and induce inflammatory responses.
The placenta also induces the secretion of proinflammatory cytokines by internalizing macrophagederived EVs. Adipose EVs regulate glucose and glycogen metabolism in the placenta explant culture, which is associated with the onset of GDM. The endothelial cell EVs released due to high glucose levels associated with GDM may induce the disruption of the fetoplacental vascular system. The onset of GDM via endothelial cell-derived EVs has been reported to activate ROS production and the MAPK1 and AKT signaling proteins (Saez et al. 2018). Thus, pregnancy disorders such as

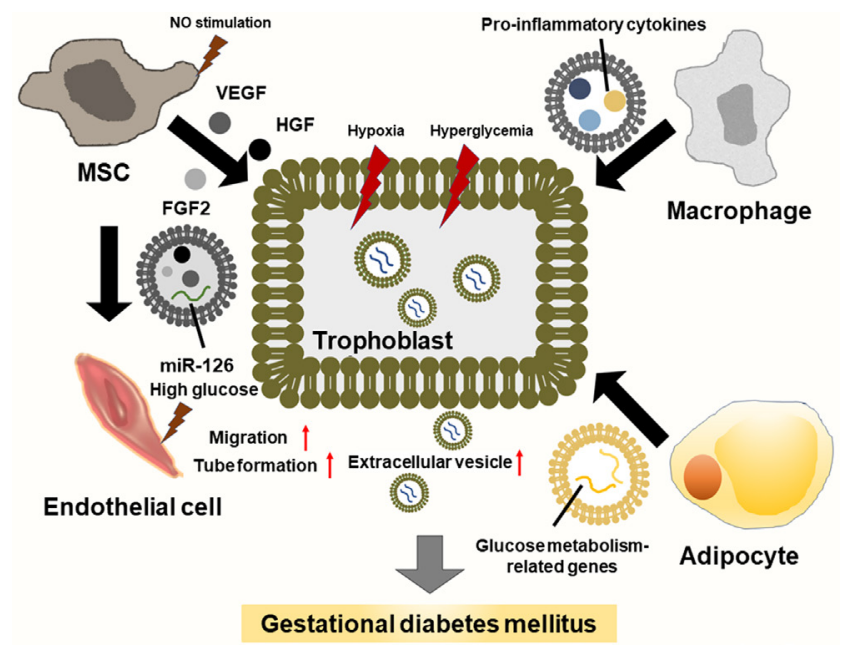

Figure 2 Hypothesis on the pathogenesis of gestational diabetes mellitus (GDM) following changes in the placental microenvironment. Under hypoxic and hyperglycemic conditions, the concentration of trophoblast EVs increases. MSCs increase endothelial cell migration through the delivery of miR-126 under high glucose conditions. Moreover, adipocytes release EVs containing transcripts related to glucose and glycogen metabolism. Pro-inflammatory cytokines in macrophage-derived EVs also induce the onset of GDM in the placental microenvironment.
GDM can be caused by interactions of various types of cells, including trophoblast cells, in the placental microenvironment (Fig. 2).

\section{Diagnosis of pregnancy disease using EVs}

The ratio of placenta-specific EVs to total circulating EVs was reported to be positively correlated with fetal growth. Several studies have suggested that placental miRNAs may be involved in fetal growth restriction. Cohort studies show that miRNAs in the serum EVs of pregnant women in their second trimester can also help predict fetal growth (Rodosthenous et al. 2017). In addition to predicting fetal growth, much evidence suggests that placental EVs can be used to diagnose pregnancy disorders.

\section{Pre-eclampsia}

$\mathrm{PE}$, characterized by hypertension and proteinuria after 20 weeks of gestation, adversely affects both the mother and fetus, exhibiting symptoms such as kidney and liver dysfunction and prematurity. PE is caused by abnormal placental development and is difficult to diagnose early due to insufficient understanding of its molecular pathogenesis mechanism. In a previous study, concentrations of placenta-specific EVs were clinically correlated to distinguish between normal pregnancy, early onset of PE and late onset of PE (Pillay et al. 2016). The expression of circulating miRNAs was also reported to be altered in the body fluids of PE patients when compared to controls. Notably, miR-885-5p exhibited higher expression than apoptotic bodies or microvesicles in the plasma-derived EV-rich fractions of PE patients. Moreover, miR-136, -494 and -495 in the EVs secreted by umbilical cord mesenchymal stem cells (UCMSCs) were also highly expressed in PE patients 
when compared to healthy controls. The expression of miR-210 was found to be higher in PE placenta when compared to normal placenta; coincidentally, hypoxic conditions induce a high level of miR-210 release via EVs (Biro et al. 2019).

Proteins present in placental EVs can also be diagnostic markers due to their altered expression in pregnant women with PE when compared to that in normal pregnant women. The FLT1 binds to VEGF and interferes with vascular homeostasis. Levels of sFlt-1 in placenta-derived EVs are higher in patients with severe PE. Moreover, plasma-derived EVs of PE patients contain a large amount of FLT1 and endoglin (ENG), which cause vascular dysfunction when administered to pregnant mice (Chang et al. 2018). Proteomic analysis using umbilical cord blood-derived EVs revealed 29 instances of significantly different protein expression between PE patients and the control group (Jia et al. 2015). It is also likely that EVs isolated from urine can be used in the diagnosis of PE (Nielsen et al. 2017). Syncytin-2 expression, which was found to be reduced in the serum EVs of PE patients, is important for the cell uptake of EVs (Vargas et al. 2014). Galectin 13 (LGALS13) is one of the galectins that is specifically expressed in the placenta. The reduction of LGALS13 level in placental tissue and placenta-derived EVs is also associated with PE progression (Than et al. 2014).

\section{Preterm birth}

The precise cause of PB is still unclear; however, the action of biochemicals released by mature fetal organs is suspected to cause an inflammatory response that interferes with the maintenance of pregnancy. EVs derived from UCMSCs in term or preterm infants have been shown to have different oxidative metabolism (Panfoli et al. 2016). This suggests that the different effects of EVs on aerobic respiration by gestational age may affect the onset of preterm delivery. EVs derived from the milk of women who underwent PB survived even after gastric and pancreatic digestion, and miRNAs in EVs displayed different expression patterns when compared to same from mothers who underwent term birth (TB) (Kahn et al. 2018). Recent studies have shown that there are 173 different miRNAs in EVs between PB and TB, and bioinformatic analysis suggests that these miRNAs target TGFB1 and TP53 signaling (Menon et al. 2019). Various circulating microparticle proteins extracted from the plasma of 10- to 12-week pregnant women show correlation with PB. Moreover, evidence suggests that miRNA in the EVs of plasma can act as a biomarker to predict PB (Fallen et al. 2018). Complex genetic networks, including PI3K/AKT and VEGF signaling, regulated by miRNAs in EVs are assumed to be involved in the onset of PB. Furthermore, cytoscape analysis of the UCMSCs of PB and TB infants revealed a difference between inflammation and metabolic cluster between the two groups (Bruschi et al. 2018).

\section{Gestational diabetes mellitus}

GDM affects 9 15\% of all pregnancies and is screened according to oral glucose tolerance tests at 24-28 weeks of gestation (Chu et al. 2007). An earlier diagnosis of GDM would allow for faster response to the damage accumulated by glucose intolerance. Hyperglycemia increases the release of EVs from primary trophoblast cells, which is further enhanced under hypoxic conditions (Rice et al. 2015). According to a cohort study, both normal and GDM pregnancies show a greater increase in the concentration of placenta-derived $\mathrm{EVs}$ as pregnancy progresses; however, a larger increase can be observed in GDM pregnancies (Salomon et al. 2016). The plasma EVs of pregnant women with GDM further promote the release of inflammatory cytokines from endothelial cells. Placental EVs from GDM pregnancy also show different miRNA expression patterns when compared to normal EVs and are associated with skeletal muscle insulin sensitivity. Notably, placental EVs in normal pregnant women increase glucose uptake in the diabetic skeletal muscle (Nair et al. 2018).

\section{The effect of EVs on livestock pregnancy}

In livestock, the endometrium secretes numerous soluble substances that are responsible for the growth and elongation of the conceptus, which is abundant in the EVs. Pregnancy loss occurs frequently during blastocyst hatching and conceptus implantation onto the endometrium; however, the factors responsible for intercellular interactions are still not completely understood. Even in livestock, placental EVs likely play an important role in maintaining pregnancy (Table 2 ). Several evidences suggest that the endometrium and placenta release microvesicles, including exosomes, to induce conceptus-endometrial interactions via the uterine luminal fluid (ULF). EVs isolated from the ULF of pregnant ewes show many differences in protein and miRNA profiles in EVs when compared to cyclic ULFs. Furthermore, EVs identified in the ULF of pregnant ewes contain endogenous beta retrovirus (enJSRV) RNA, which can be delivered to heterologous cells (Burns et al. 2014). Exosomes containing enJSRV protein also promote the proliferation and the secretion of interferon tau (IFNT) on ovine trophectoderm cells (Ruiz-Gonzalez et al. 2015). Moreover, EVs labeled with PKH67 dye were observed in both the trophectoderm and uterine epithelium (Burns et al. 2016). This suggests that the cell-to-cell interactions via EVs play an important role in maintaining normal pregnancy in sheep. Additionally, EVs isolated from the uterine flushings of pregnant ewes had proteins such as IFNT, macrophage-capping protein 
Table 2 Effect of EVs on livestock pregnancy.

\begin{tabular}{llll}
\hline Species/EV source & Biological functions & Bioactive substances in EVs & Reference \\
\hline $\begin{array}{l}\text { Sheep } \\
\text { Uterine luminal fluid }\end{array}$ & $\begin{array}{l}\text { Conceptus-endometrial interactions } \\
\text { Uterine flushing }\end{array}$ & $\begin{array}{l}\text { enJSRVs RNA } \\
\text { enJSRVs RNA }\end{array}$ & Burns et al. 2014 \\
$\begin{array}{l}\text { Pig } \\
\text { Trophectoderm cells }\end{array}$ & Angiog-Gonzalez et al. 2015 \\
$\begin{array}{l}\text { Umbilical cord blood } \\
\text { Cow }\end{array}$ & Angiogenic regulation & Angiogenic miRNAs & Bidarimath et al. 2017 \\
$\begin{array}{l}\text { Uterus } \\
\text { Uterine flushing }\end{array}$ & Development of SCNT embryos & miR-15 & Luo et al. 2018 \\
Peripheral blood & Genetic regulation & - & Qiao et al. 2018 \\
\hline
\end{tabular}

(CAPG), and aldo-keto reductase family 1 , member B1 protein (AKR1B1), which are not found in the EVs of the cyclic ewes.

In porcine pregnancies, placentation begins with the expression of adherent molecules in the trophectoderm and uterine luminal epithelium over days 15-20 during the gestation period. The expression of EV-related genes in the porcine uterus shows a specific difference depending on the cell type. The EVs derived from porcine trophectoderm cells induce the proliferation of the maternal endothelial cells and promote angiogenic processes (Bidarimath et al. 2017). The expression of porcine miR-150 is reduced in the umbilical cord blood-derived EVs of pigs undergoing IUGR (Luo et al. 2018). The increase in miR-150 in the EVs increases the proliferation and migration of endothelial cells, thereby exhibiting a pro-angiogenic effect.

When bovine EVs isolated from a luteal phase uterus were supplemented with bovine somatic cell nuclear transfer (SCNT) embryos, the blastocyst formation rate and inner cell mass/trophectoderm cell ratio were observed to increase (Qiao et al. 2018). This suggests that EVs released from bovine uteri may play an important role in embryo development. Treatment of EVs from the flushed fluids of a bovine uterus during pregnancy (at days 17,20, and 22) leads to differences in the expression of apoptosis-related genes and adhesion molecules in endometrial epithelial cells depending on the gestational period (Kusama et al. 2018). Bovine placenta-specific EVs inhibit the activation of NF- $\mathrm{BB}$ via miR-499 transfer to endometrial epithelial cells and also inhibit the inflammatory response induced by LPS (Zhao et al. 2018).

\section{Conclusion}

The placenta during early pregnancy is in an environment of mild inflammation and hypoxia, and normal proliferation and invasion of trophoblasts is necessary for the maintenance of pregnancy. Various miRNAs and proteins in placenta-derived EVs are known to play a role in pregnancy maintenance in the trophoblast and placental microenvironment. Therefore, understanding the physiological activity of EVs during early pregnancy will present a new approach to overcome abnormal placentation and pregnancy disorders.

\section{Declaration of interest}

The authors declare that there is no conflict of interest that could be perceived as prejudicing the impartiality of this review.

\section{Funding}

This research was supported by a grant of the Korea Health Technology R\&D Project through the Korea Health Industry Development Institute funded by the Ministry of Health \& Welfare (grant number: HI17C0929) and the National Research Foundation of Korea (NRF) grant funded by the Ministry of Science and ICT (MSIT) (Grant number: 2018R1C1B6009048).

\section{Author contribution statement}

G S and W L designed and directed the study. C Y, G S and W L wrote and prepared the manuscript. C Y designed the figures. All authors provided critical feedback and helped to shape the manuscript.

\section{References}

Atay S, Gercel-Taylor C, Suttles J, Mor G \& Taylor DD 2011a Trophoblastderived exosomes mediate monocyte recruitment and differentiation. American Journal of Reproductive Immunology 65 65-77. (https://doi. org/10.1111/j.1600-0897.2010.00880.x)

Atay S, Gercel-Taylor C \& Taylor DD 2011b Human trophoblast-derived exosomal fibronectin induces pro-inflammatory IL-1 beta production by macrophages. American Journal of Reproductive Immunology $\mathbf{6 6}$ 259-269. (https://doi.org/10.1111/j.1600-0897.2011.00995.x)

Bidarimath M, Khalaj K, Kridli RT, Kan FWK, Koti M \& Tayade C 2017 Extracellular vesicle mediated intercellular communication at the porcine maternal-fetal interface: a new paradigm for conceptusendometrial cross-talk. Scientific Reports 7 40476. (https://doi. org/10.1038/srep40476)

Biro O, Fothi Á, Alasztics B, Nagy B, Orban TI \& Rigo J, Jr 2019 Circulating exosomal and Argonaute-bound microRNAs in preeclampsia. Gene $\mathbf{6 9 2}$ 138-144. (https://doi.org/10.1016/j.gene.2019.01.012)

Boriachek K, Masud MK, Palma C, Phan HP, Yamauchi Y, Hossain MSA, Nguyen NT, Salomon C \& Shiddiky MJA 2019 Avoiding pre-isolation step in exosome analysis: direct isolation and sensitive detection of exosomes using gold-loaded nanoporous ferric oxide nanozymes. Analytical Chemistry 91 3827-3834. (https://doi.org/10.1021/acs. analchem.8b03619) 
Bruschi M, Santucci L, Ravera S, Bartolucci M, Petretto A, Calzia D, Ghiggeri GM, Ramenghi LA, Candiano G \& Panfoli I 2018 Metabolic signature of microvesicles from umbilical cord mesenchymal stem cells of preterm and term infants. Proteomics: Clinical Applications 12 e1700082. (https://doi.org/10.1002/prca.201700082)

Burns G, Brooks K, Wildung M, Navakanitworakul R, Christenson LK \& Spencer TE 2014 Extracellular vesicles in luminal fluid of the ovine uterus. PLOS ONE 9 e90913. (https://doi.org/10.1371/journal. pone.0090913)

Burns GW, Brooks KE \& Spencer TE 2016 Extracellular vesicles originate from the conceptus and uterus during early pregnancy in sheep. Biology of Reproduction 94 56. (https://doi.org/10.1095/biolreprod.115.134973)

Chang X, Yao J, He Q, Liu M, Duan T \& Wang K 2018 Exosomes from women with preeclampsia induced vascular dysfunction by delivering sFlt (soluble Fms-like tyrosine kinase)- 1 and sEng (soluble endoglin) to endothelial cells. Hypertension 72 1381-1390. (https://doi.org/10.1161/ HYPERTENSIONAHA.118.11706)

Chu SY, Callaghan WM, Kim SY, Schmid CH, Lau J, England LJ \& Dietz PM 2007 Maternal obesity and risk of gestational diabetes mellitus. Diabetes Care 30 2070-2076. (https://doi.org/10.2337/dc06-2559a)

Delorme-Axford E, Donker RB, Mouillet JF, Chu T, Bayer A, Ouyang Y, Wang T, Stolz DB, Sarkar SN, Morelli AE et al. 2013 Human placental trophoblasts confer viral resistance to recipient cells. PNAS 110 12048-12053. (https://doi.org/10.1073/pnas.1304718110)

Donker RB, Mouillet JF, Chu T, Hubel CA, Stolz DB, Morelli AE \& Sadovsky Y 2012 The expression profile of C19MC microRNAs in primary human trophoblast cells and exosomes. Molecular Human Reproduction 18 417-424. (https://doi.org/10.1093/molehr/gas013)

Du W, Zhang K, Zhang S, Wang R, Nie Y, Tao H, Han Z, Liang L, Wang D, Liu J et al. 2017 Enhanced proangiogenic potential of mesenchymal stem cell-derived exosomes stimulated by a nitric oxide releasing polymer. Biomaterials 133 70-81. (https://doi.org/10.1016/j. biomaterials.2017.04.030)

Elfeky O, Longo S, Lai A, Rice GE \& Salomon C 2017 Influence of maternal $\mathrm{BMI}$ on the exosomal profile during gestation and their role on maternal systemic inflammation. Placenta 50 60-69. (https://doi.org/10.1016/j. placenta.2016.12.020)

Fallen S, Baxter D, Wu X, Kim TK, Shynlova O, Lee MY, Scherler K, Lye S, Hood L \& Wang K 2018 Extracellular vesicle RNAs reflect placenta dysfunction and are a biomarker source for preterm labour. Journal of Cellular and Molecular Medicine 22 2760-2773. (https://doi. org/10.1111/jcmm.13570)

Greening DW, Nguyen HP, Elgass K, Simpson RJ \& Salamonsen LA 2016 Human endometrial exosomes contain hormone-specific cargo modulating trophoblast adhesive capacity: insights into endometrialembryo interactions. Biology of Reproduction 94 38. (https://doi. org/10.1095/biolreprod.115.134890)

Gupta AK, Holzgreve W \& Hahn S 2008 Decrease in lipid levels of syncytiotrophoblast micro-particles reduced their potential to inhibit endothelial cell proliferation. Archives of Gynecology and Obstetrics 277 115-119. (https://doi.org/10.1007/s00404-007-0425-2)

Gysler SM, Mulla MJ, Guerra M, Brosens JJ, Salmon JE, Chamley LW \& Abrahams VM 2016 Antiphospholipid antibody-induced miR-146a-3p drives trophoblast interleukin-8 secretion through activation of toll-like receptor 8. Molecular Human Reproduction 22 465-474. (https://doi. org/10.1093/molehr/gaw027)

Hedlund M, Stenqvist AC, Nagaeva O, Kjellberg L, Wulff M, Baranov V \& Mincheva-Nilsson L 2009 Human placenta expresses and secretes NKG2D ligands via exosomes that down-modulate the cognate receptor expression: evidence for immunosuppressive function. Journal of Immunology 183 340-351. (https://doi.org/10.4049/jimmunol.0803477)

Jauniaux E, Gulbis B \& Burton GJ 2003 Physiological implications of the materno-fetal oxygen gradient in human early pregnancy. Reproductive Biomedicine Online 7 250-253. (https://doi.org/10.1016/S14726483(10)61760-9)

Jia R, Li J, Rui C, Ji H, Ding H, Lu Y, De W \& Sun L 2015 Comparative proteomic profile of the human umbilical cord blood exosomes between normal and preeclampsia pregnancies with high-resolution mass spectrometry. Cellular Physiology and Biochemistry 36 2299-2306. (https://doi.org/10.1159/000430193)

Kahn S, Liao Y, Du X, Xu W, Li J \& Lonnerdal B 2018 Exosomal microRNAs in milk from mothers delivering preterm infants survive in vitro digestion and are taken up by human intestinal cells. Molecular Nutrition and Food Research 62 e1701050. (https://doi.org/10.1002/mnfr.201701050) Kambe S, Yoshitake H, Yuge K, Ishida Y, Ali MM, Takizawa T, Kuwata T, Ohkuchi A, Matsubara S, Suzuki M et al. 2014 Human exosomal placenta-associated miR-517a-3p modulates the expression of PRKG1 mRNA in Jurkat cells. Biology of Reproduction 91 129. (https://doi. org/10.1095/biolreprod.114.121616)

Kong P, Xie X, Li F, Liu Y \& Lu Y 2013 Placenta mesenchymal stem cell accelerates wound healing by enhancing angiogenesis in diabetic Goto-Kakizaki (GK) rats. Biochemical and Biophysical Research Communications 438 410-419. (https://doi.org/10.1016/j. bbrc.2013.07.088)

Kusama K, Nakamura K, Bai R, Nagaoka K, Sakurai T \& Imakawa K 2018 Intrauterine exosomes are required for bovine conceptus implantation. Biochemical and Biophysical Research Communications 495 1370-1375. (https://doi.org/10.1016/j.bbrc.2017.11.176)

Lotvall J, Hill AF, Hochberg F, Buzas El, Di Vizio D, Gardiner C, Gho YS, IV, Kurochkin IV, Mathivanan S, Quesenberry P et al. 2014 Minimal experimental requirements for definition of extracellular vesicles and their functions: a position statement from the International Society for extracellular vesicles. Journal of Extracellular Vesicles 3 26913. (https:// doi.org/10.3402/jev.v3.26913)

Luo J, Fan Y, Shen L, Niu L, Zhao Y, Jiang D, Zhu L, Jiang A, Tang Q, Ma J et al. 2018 The pro-angiogenesis of exosomes derived From umbilical cord blood of intrauterine growth restriction pigs was repressed associated with MiRNAs. International Journal of Biological Sciences 14 1426-1436. (https://doi.org/10.7150/ijbs.27029)

Menon R, Debnath C, Lai A, Guanzon D, Bhatnagar S, Kshetrapal PK, Sheller-Miller S, Salomon C \& Garbhini Study Team 2019 Circulating exosomal miRNA profile during term and preterm birth pregnancies: a longitudinal study. Endocrinology 160 249-275. (https://doi. org/10.1210/en.2018-00836)

Mincheva-Nilsson L, Nagaeva O, Chen T, Stendahl U, Antsiferova J, Mogren I, Hernestal J \& Baranov V 2006 Placenta-derived soluble MHC class I chain-related molecules down-regulate NKG2D receptor on peripheral blood mononuclear cells during human pregnancy: a possible novel immune escape mechanism for fetal survival. Journal of Immunology 176 3585-3592. (https://doi.org/10.4049/ jimmunol.176.6.3585)

Motta-Mejia C, Kandzija N, Zhang W, Mhlomi V, Cerdeira AS, Burdujan A, Tannetta D, Dragovic R, Sargent IL, Redman CW et al. 2017 Placental vesicles carry active endothelial nitric oxide synthase and their activity is reduced in preeclampsia. Hypertension 70 372-381. (https://doi. org/10.1161/HYPERTENSIONAHA.117.09321)

Nair S, Jayabalan N, Guanzon D, Palma C, Scholz-Romero K, Elfeky O, Zuniga F, Ormazabal V, Diaz E, Rice GE et al. 2018 Human placental exosomes in gestational diabetes mellitus carry a specific set of miRNAs associated with skeletal muscle insulin sensitivity. Clinical Science 132 2451-2467. (https://doi.org/10.1042/CS20180487)

Nielsen MR, Frederiksen-Moller B, Zachar R, Jorgensen JS, Hansen MR, Ydegaard R, Svenningsen P, Buhl K \& Jensen BL 2017 Urine exosomes from healthy and hypertensive pregnancies display elevated level of alpha-subunit and cleaved alpha- and gamma-subunits of the epithelial sodium channel-ENaC. Pflugers Archiv 469 1107-1119. (https://doi. org/10.1007/s00424-017-1977-z)

Niu Z, Pang RTK, Liu W, Li Q, Cheng R \& Yeung WSB 2017 Polymer-based precipitation preserves biological activities of extracellular vesicles from an endometrial cell line. PLOS ONE 12 e0186534. (https://doi. org/10.1371/journal.pone.0186534)

Panfoli I, Ravera S, Podesta M, Cossu C, Santucci L, Bartolucci M, Bruschi M, Calzia D, Sabatini F, Bruschettini M et al. 2016 Exosomes from human mesenchymal stem cells conduct aerobic metabolism in term and preterm newborn infants. FASEB Journal 30 1416-1424. (https://doi.org/10.1096/fj.15-279679)

Petroff MG, Kharatyan E, Torry DS \& Holets L 2005 The immunomodulatory proteins B7-DC, B7-H2, and B7-H3 are differentially expressed across gestation in the human placenta. American Journal of Pathology 167 465-473. (https://doi.org/10.1016/S0002-9440(10)62990-2)

Pillay P, Maharaj N, Moodley J \& Mackraj I 2016 Placental exosomes and pre-eclampsia: maternal circulating levels in normal pregnancies and, early and late onset pre-eclamptic pregnancies. Placenta 46 18-25. (https://doi.org/10.1016/j.placenta.2016.08.078) 
Qiao F, Ge H, Ma X, Zhang Y, Zuo Z, Wang M, Zhang Y \& Wang Y 2018 Bovine uterus-derived exosomes improve developmental competence of somatic cell nuclear transfer embryos. Theriogenology 114 199-205. (https://doi.org/10.1016/j.theriogenology.2018.03.027)

Redman CW \& Sargent IL 2007 Microparticles and immunomodulation in pregnancy and pre-eclampsia. Journal of Reproductive Immunology $\mathbf{7 6}$ 61-67. (https://doi.org/10.1016/j.jri.2007.03.008)

Rice GE, Scholz-Romero K, Sweeney E, Peiris H, Kobayashi M, Duncombe G, Mitchell MD \& Salomon C 2015 The effect of glucose on the release and bioactivity of exosomes from first trimester trophoblast cells. Journal of Clinical Endocrinology and Metabolism 100 E1280-E1288. (https://doi.org/10.1210/jc.2015-2270)

Rodosthenous RS, Burris HH, Sanders AP, Just AC, Dereix AE, Svensson K, Solano M, Tellez-Rojo MM, Wright RO \& Baccarelli AA 2017 Second trimester extracellular microRNAs in maternal blood and fetal growth: an exploratory study. Epigenetics 12 804-810. (https://doi.org/10.1080/ 15592294.2017.1358345)

Ruiz-Gonzalez I, Xu J, Wang X, Burghardt RC, Dunlap KA \& Bazer FW 2015 Exosomes, endogenous retroviruses and toll-like receptors: pregnancy recognition in ewes. Reproduction 149 281-291. (https://doi. org/10.1530/REP-14-0538)

Sabapatha A, Gercel-Taylor C \& Taylor DD 2006 Specific isolation of placenta-derived exosomes from the circulation of pregnant women and their immunoregulatory consequences. American Journal of Reproductive Immunology 56 345-355. (https://doi.org/10.1111/j.16000897.2006.00435.x)

Saez T, Salsoso R, Leiva A, Toledo F, de Vos P, Faas M \& Sobrevia L 2018 Human umbilical vein endothelium-derived exosomes play a role in foetoplacental endothelial dysfunction in gestational diabetes mellitus. Biochimica et Biophysica Acta: Molecular Basis of Disease 1864 499-508. (https://doi.org/10.1016/j.bbadis.2017.11.010)

Salomon C, Scholz-Romero K, Sarker S, Sweeney E, Kobayashi M, Correa P, Longo S, Duncombe G, Mitchell MD, Rice GE et al. 2016 Gestational diabetes mellitus is associated with changes in the concentration and bioactivity of placenta-derived exosomes in maternal circulation across gestation. Diabetes 65 598-609. (https:// doi.org/10.2337/db15-0966)

Sargent IL, Germain SJ, Sacks GP, Kumar S \& Redman CW 2003 Trophoblast deportation and the maternal inflammatory response in preeclampsia. Journal of Reproductive Immunology 59 153-160. (https:// doi.org/10.1016/S0165-0378(03)00044-5)

Sarker S, Scholz-Romero K, Perez A, Illanes SE, Mitchell MD, Rice GE \& Salomon C 2014 Placenta-derived exosomes continuously increase in maternal circulation over the first trimester of pregnancy. Journal of Translational Medicine 12 204. (https://doi.org/10.1186/1479-5876-12204)

Sheller-Miller S, Lei J, Saade G, Salomon C, Burd I \& Menon R 2016 Fetomaternal trafficking of exosomes in murine pregnancy models. Frontiers in Pharmacology 7 432. (https://doi.org/10.3389/fphar.2016.00432)

Sheller-Miller S, Trivedi J, Yellon SM \& Menon R 2019 Exosomes cause preterm birth in mice: evidence for paracrine signaling in pregnancy. Scientific Reports 9 608. (https://doi.org/10.1038/s41598-018-37002-x)

Shen L, Li Y, Li R, Diao Z, Yany M, Wu M, Sun H, Yan G \& Hu Y 2018 Placentaassociated serum exosomal miR155 derived from patients with preeclampsia inhibits eNOS expression in human umbilical vein endothelial cells. International Journal of Molecular Medicine $4 \mathbf{1}$ 1731-1739. (https://doi.org/10.3892/ijmm.2018.3367)

Stenqvist AC, Nagaeva O, Baranov V \& Mincheva-Nilsson L 2013 Exosomes secreted by human placenta carry functional Fas ligand and TRAIL molecules and convey apoptosis in activated immune cells, suggesting exosome-mediated immune privilege of the fetus. Journal of Immunology 191 5515-5523. (https://doi.org/10.4049/jimmunol.1301885)

Southcombe J, Tannetta D, Redman C \& Sargent I 2011 The immunomodulatory role of syncytiotrophoblast microvesicles. PLoS One 6 e20245. (https://doi.org/10.1371/journal.pone.0020245)

Takahashi H, Ohkuchi A, Kuwata T, Usui R, Baba Y, Suzuki H, Chaw Kyi TT, Matsubara S, Saito S \& Takizawa T 2017 Endogenous and exogenous miR-520c-3p modulates CD44-mediated extravillous trophoblast invasion. Placenta 50 25-31. (https://doi.org/10.1016/j. placenta.2016.12.016)

Than NG, Balogh A, Romero R, Karpati E, Erez O, Szilagyi A, Kovalszky I, Sammar M, Gizurarson S, Matko J et al. 2014 Placental protein 13 (PP13) - a placental immunoregulatory galectin protecting pregnancy. Frontiers in Immunology 5 348. (https://doi.org/10.3389/fimmu.2014.00348)

Tolosa JM, Schjenken JE, Clifton VL, Vargas A, Barbeau B, Lowry P, Maiti K \& Smith R 2012 The endogenous retroviral envelope protein syncytin-1 inhibits LPS/PHA-stimulated cytokine responses in human blood and is sorted into placental exosomes. Placenta 33 933-941. (https://doi. org/10.1016/j.placenta.2012.08.004)

Tong M, Kleffmann T, Pradhan S, Johansson CL, DeSousa J, Stone PR, James JL, Chen Q \& Chamley LW 2016 Proteomic characterization of macro-, micro-and nano-extracellular vesicles derived from the same first trimester placenta: relevance for feto-maternal communication. Human Reproduction 31 687-699. (https://doi.org/10.1093/humrep/ dew004)

Tong M, Stanley JL, Chen Q, James JL, Stone PR \& Chamley LW 2017 Placental nano-vesicles target to specific organs and modulate vascular tone in vivo. Human Reproduction 32 2188-2198. (https://doi. org/10.1093/humrep/dex310)

Vargas A, Zhou S, Ethier-Chiasson M, Flipo D, Lafond J, Gilbert C \& Barbeau B 2014 Syncytin proteins incorporated in placenta exosomes are important for cell uptake and show variation in abundance in serum exosomes from patients with preeclampsia. FASEB Journal $\mathbf{2 8}$ 3703-3719. (https://doi.org/10.1096/fj.13-239053)

Vickers KC, Palmisano BT, Shoucri BM, Shamburek RD \& Remaley AT 2011 MicroRNAs are transported in plasma and delivered to recipient cells by high-density lipoproteins. Nature Cell Biology 13 423-433. (https://doi.org/10.1038/ncb2210)

Vilella F, Moreno-Moya JM, Balaguer N, Grasso A, Herrero M, Martinez S, Marcilla A \& Simon C 2015 Hsa-miR-30d, secreted by the human endometrium, is taken up by the pre-implantation embryo and might modify its transcriptome. Development 142 3210-3221. (https://doi. org/10.1242/dev.124289)

Wang Z, Wang P, Wang Z, Qin Z, Xiu X, Xu D, Zhang X \& Wang Y 2019 MiRNA-548c-5p downregulates inflammatory response in preeclampsia via targeting PTPRO. Journal of Cellular Physiology 234 11149-11155. (https://doi.org/10.1002/jcp.27758)

Zhao G, Yang C, Yang J, Liu P, Jiang K, Shaukat A, Wu H \& Deng G 2018 Placental exosome-mediated Bta-miR-499-Lin28B/let-7 axis regulates inflammatory bias during early pregnancy. Cell Death and Disease 9 704. (https://doi.org/10.1038/s41419-018-0713-8)

Received 28 March 2019

First decision 9 May 2019

Revised manuscript received 18 June 2019

Accepted 25 June 2019 